NASA TECHNICAL NOTE

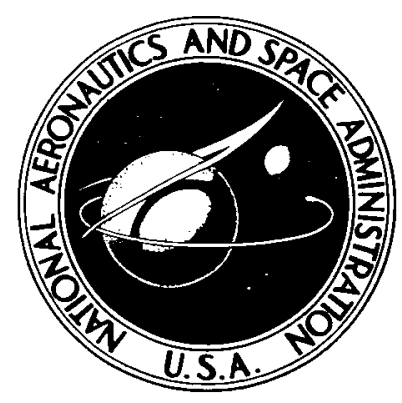

NASA TN D-3198

$\infty$
$\frac{0}{a}$
$\vdots$
$z$
$\vdots$
$\vdots$
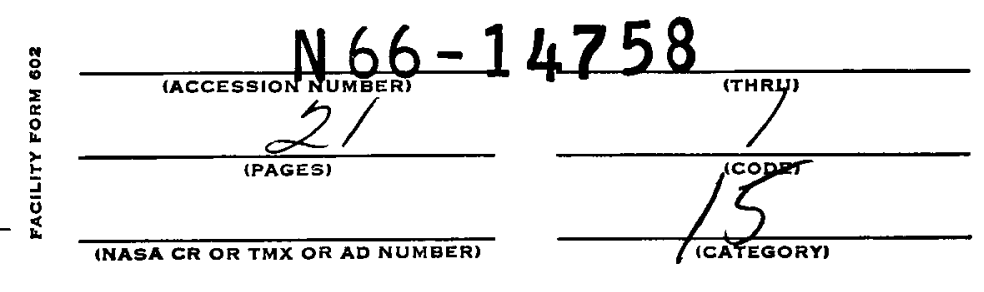

DYNAMIC INSTABILITY OF

UNDAMPED BELLOWS FACE SEALS

IN CRYOGENIC LIQUID

by Jobn C. Hudelson

Lewis Research Center

Cleveland, Obio
GPO PRICE

CFSTI PRICE(S)

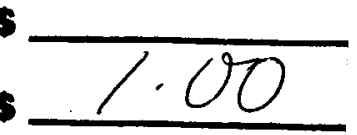

Hard copy (HC)

Microfiche (MF)

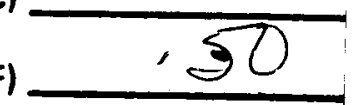

ก 053 July 65

NATIONAL AERONAUTICS AND SPACE ADMINISTRATION - WASHINGTON, D. C. - JANUARY 1966 


\section{DYNAMIC INSTABILITY OF UNDAMPED BELLOWS FACE SEALS IN CRYOGENIC LIQUID}

By John C. Hudelson

Lewis Research Center

Cleveland, Ohio

\section{NATIONAL AERONAUTICS AND SPACE ADMINISTRATION}


DYNAMIC INSTABILITY OF UNDAMPED BELLOWS FACE SEAIS IN CRYOGENIC LIQUID

by John C. Hudelson

Lewis Research Center

\section{SUMMARY}

14758

The primary motions of an undamped bellows face seal are described along with the displacements and frequencies of the motions experienced during dynamic instability.

Subminiature accelerometers were attached to the nosepiece of a $1 \frac{3}{4}$-inch nominal shaft diameter bellows seal and the accelerations and frequencies recorded on magnetic tape for analysis at an expanded time factor.

The results of the tests indicated that dynamic instability will occur in undamped bellows face seals operating in a cryogenic environment and be of such a magnitude as to damage the sealing surface causing excessive leakage. Variations in the pressure difference across the seal and in the contact load between the seal nosepiece and the mating surface did not change the characteristics of the dynamic instability. The primary motions of dynamic instability are diametral rocking and torsional oscillation.

\section{INTIRODUCTION}

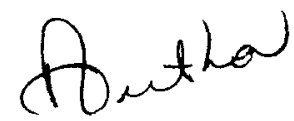

Investigators in the field of dynamic sealing devices agree that bellowstype face seals are subject to dynamic instabilities (refs. 1 and 2). Bellows face seals assumed to be operating in an unstable manner suffer mechanical damage and show poor operating characteristics such as short life, excessive leakage rates, and noise generation (ref. 2).

Theoretical treatment of the dynamics of bellows seal operation (ref. 3) does not consider the effects of friction forces, stick slip phenomena, and phase change of the sealed fluid in the interface. Mating face runout and shaft axial motion are assumed to be the primary exciting forces of bellows seal instability. Under these assumptions the minimum force necessary to maintain contact between the seal nosepiece and the mating face under various conditions of mating face runout and axial motion can be calculated.

In most seal applications the contact force is two to four times greater than the minimum contact force suggested by theory. It is also assumed that the operating frequency should be lower than the natural frequency of the bellows 
seal. Since the conditions of adequate contact load and operation below the bellows seals natural frequency are met in many applications and dynamic instability is still encountered, additional factors must be considered.

The objective of this investigation was to study modes of vibrational instability of bellows face seals operating in a cryogenic environment.

The cryogenic environment was chosen because of the number of problems that have occurred in cryogenic turbopumps that could have been caused by dynamic instability of the bellows face seals.

The test method employed subminiature accelerometers mounted on an undamped bellows face seal with a $1 \frac{3}{4}$-inch nominal shaft diameter ( 2 -in. mean sealing diam). The accelerometer data in the form of acceleration and frequency were reduced to displacements and phase relations depicting the motions of the seal.

\section{APPARATUS AIVD PROCEDURE}

\section{Test Apparatus}

The cryogenic-oxidant seal test facility at the NASA Lewis Research Center was used for this investigation. The test facility can accommodate radial face seals to nominal 3 -inch diameters. The test bellows face seals were nominally $1 \frac{3}{4}$ inch in size having a 2 -inch mean sealing diameter and did not use any damping devices such as lugs, lips, or springs. A schematic diagram of the test head (fig. 1) shows the major elements of the apparatus. The pot contains the test fluid, in this case, liquid nitrogen. One end of the pot was the adapter plate, which contained the test bellows seal. With this configuration, the seal was pressurized on the inside diameter and the leakage direction was from the inside to the outside diameter of the seal nosepiece.

During operation a continuous flow of liquid nitrogen was maintained through the pot. The liquid nitrogen was supplied to the pot near the bottom, and the gaseous nitrogen boiloff and excess liquid nitrogen were vented from the top of the pot. The fluid level was indicated on a capacitance recording device by means of a capacitance pickup in the pot.

The pot was constrained radially and torsionally but was free to move axially over a 0.200 -inch range. A screw for axially adjusting the pot had micrometer-type markings in 0.001 -inch increments. The axial position of the pot was indicated by two preset electrical contacts. These contacts were so located as to minimize the effect of contraction of the pot as it was chilled down for a test.

The desired seal deflection was obtained by first establishing the point where leakage would just start under zero shaft speed and a seal pressure of 5 pounds per square inch. Next an adjustment was made to apply the desired operating deflection. 
Surrounding the pot was an insulated spill chamber, which collected the leakage past the test seal. By purging the spill chamber with dry nitrogen gas, frost buildup was prevented. The purge rate was measured and kept constant. The gas leaving the spill chamber had two components, seal leakage and purge flow. By subtracting the purge flow, the leakage rate of the seal could be determined. The spill chamber vent gases were routed through a heat exchanger and measured at room temperature and pressure.

The drive system consisted of a 15-horsepower variable-speed direct-current motor driving a gear box with a ratio of 1 to 10 . The gear box drove a precision spindle that had a secondary shaft seal and a purge system. The rig was capable of operating at a shaft speed of $20000 \mathrm{rpm}$.

\section{Instrumentation System}

The instrumentation system used to detect and record the vibrations of the bellows seal nosepiece used self-generating piezoelectric accelerometers as the primary transducers. The accelerometers fed an alternating-current decade isolation amplifier, which in turn fed an instrumentation magnetic tape recorder.

The accelerometers used (0.225-in. diam by 0.245-in. length) were cataloged as subminiature. The head weight was 0.8 gram and were mounted by cementing. The resonant frequency was $70 \mathrm{kcps}$ and the dynamic range extended to $10000 \mathrm{~g}$. The calibrated output was 1.20 millivolts per $\mathrm{g}$.

A seven-channel instrumentation magnetic tape recorder was used to store the outputs from the four accelerometers. In addition, one channel was used for timing information and audio comments on the test such as amplification factors, pot pressure, and total leakage rates. The analog signals were recorded in direct mode with a frequency response of 35 to $40000 \mathrm{cps} \pm 3$ decibels at a tape speed of 15 inches per second. The accelerometer signals were monitored during the test on a four-channel oscilloscope. The advantage of recording the accelerometer outputs was in the ability to play back the information at a slower speed as weII as the ability to replay the information many times.

The accelerometer placement is illustrated in figure 2. Three of the accelerometers were attached to the seal nosepiece and the fourth was attached to the adapter plate adjacent to the seal housing. The accelerometers were sensitive along one axis only, thus the orientation was important. The accelerometers mounted at the $90^{\circ}$ and $180^{\circ}$ positions measured accelerations parallel to the axis of the seal. The accelerometer mounted at the $315^{\circ}$ position measured the torsional acceleration of the seal nosepiece. The fourth accelerometer, mounted on the adapter plate, measured the background accelerations and also acted as a control to monitor electrical interference. For this reason, the background accelerometer was operated at five times the sensitivity of the other accelerometers. In the trace photographs some low frequency wave shape can be seen in the background trace. This was 60-cycle hum in the playback system and does not represent background vibration.

The accelerometers mounted on the seal nosepiece increased the mass of the dynamic or vibrating part of the seal by about 5 percent, which would tend to 
decrease the resonant frequency. The light-weight cables attached to the accelerometers tend to damp out the vibrations rather than cause seal instability.

\section{Data Reduction}

When a data point was established, trace photographs were taken of the oscilloscope. Two photographs of each data point were taken using different oscilloscope sweep speeds to show more clearly the different vibrational frequencies. It was also possible, and very useful, to take a series of photographs by using the oscilloscope time delay. The photographs would overlap about 25 percent in each direction, and by matching and cutting them a composite photograph could be made. Figure 3 is a portion of such a photograph that was 46 inches long and covered 0.169 second of real time. Figure 3 will be discussed in detail in the section Shifting Phase Relation.

The data were obtained from the trace photographs as vibration frequencies, vector accelerations in $g^{\prime} s$, and phase relations between the different accelerometers. A slight decrease in the amplitude of the recorded accelerations occurred when they were played back at a slower tape speed. The decrease was a function of both the playback speed and the frequency of the recorded signal. In almost all cases a factor in the range of 1.04 to 1.08 was used to correct the data before determining the vibrational displacement. The double amplitude displacement was obtained from the acceleration and frequency by the following relation:

$$
\text { displacement, in. }=\frac{\text { acceleration, } g^{\prime} s}{0.0512 \times(\text { frequency, } \mathrm{cps})^{2}}
$$

\section{Test Parameters}

The other parameters affecting seal operation were measured and recorded. Each bellows seal was calibrated as to load against deflection at both room temperature and liquid nitrogen temperature $\left(-320^{\circ} \mathrm{F}\right)$. The seal nosepiece was checked for flatness at room temperature after five cycles to $-320^{\circ} \mathrm{F}$. The seal was relapped if necessary to within three sodium light bands. The seal nosepiece material was a metal-fluoride-impregnated mechanical carbon. The hard chrome plate mating surface was adjusted to run within a total indicator reading of $500 \pm 100$ microinches at the contact radius. The same seals and mating surfaces were used for a series of tests rather than using new hardware for each test. The investigation was therefore of seal instabilities for run-in seals, not new seal-mating face combinations.

Between tests the seal and the mating face were examined and the seal nosepiece was measured for wear. If any abnormal nosepiece wear was indicated, a surface profile recording was taken of the seal nosepiece. Before and after each test static room-temperature gaseous-nitrogen leakage data were taken. The bellows was pressure checked to determine if any fatigue cracks had developed. 
RESULTS

Free Vibration

The natural vibration characteristics of the bellows face seals were measured by plucking the seal nosepiece and allowing it to vibrate out of contact with the mating surface. At the onset of free vibration, high-frequency oscillations were visible in both axial and torsional directions. These highfrequency vibrations of about $20 \mathrm{kcps}$ were damped out in about 4 milliseconds. Figure 4(a) shows the high-frequency vibrations decreasing in amplitude and the emergence of medium- and low-frequency vibrations. The axial vibration frequencies were 120, 500, and $2800 \mathrm{cps}$. The torsional vibrational frequency was $3200 \mathrm{cps}$. The top trace, axial 1 accelerometer, of figure 4 (b) shows the 120and 2800-cps frequencies combined. The second trace, axial 2 accelerometer, shows the 500- and 2800-cps combined frequencies. The third trace represents the torsional accelerometer and shows the 3200-cps vibration. In all the trace photographs the abscissa scale is the same, and each large division is 0.00125 second.

Figure $4(\mathrm{c})$ shows the same excitation at a mean time from 0.0500 to 0.0625 second after initiation. The two top traces, which represent the axial accelerometers, show only the 500-cps vibration. A weak 500-cps signal in the third or torsional trace can also be seen. This has been excited by the axial vibration that predominates.

The free vibrations from the static excitation were damped out in about 0.25 second. The length of time the vibrations persist is a function of the initial excitement but approaches a limit at 0.5 second. No attempt to determine the coefficient of damping was made because of the complex nature of the vibration.

\section{Modes of Motion}

There are four predominate modes of motion of the seal nosepiece during unstable operation. The first motion would be straight axial displacement with the sealing face remaining perpendicular to the axis of the seal. The second is torsional oscillation about the seal axis.

The third motion has many complex forms. Fundamentally it is a rocking about a diameter where there would be intermittent single point contact between the seal nosepiece and the mating face. This point of contact shifts to a point diametrically opposite the initial contact point, and the nosepiece is completely out of contact during the shift or rocking. The rocking diameter is perpendicular to a line connecting the points of contact.

This diametral rocking motion takes on a more complex form when the rocking axis rotates in a plane perpendicular to the axis of the seal.

The fourth motion has been called a nutation and is similar to a spinning plate just before it comes to rest. At first the nutation appears to be a special case of diametral rocking, but it fails to satisfy the description of 
diametral rocking. In nutation the contact between the seal nosepiece and the . mating face is continuous. It is always changing but is in constant contact, while in diametral rocking the contact is discontinuous.

\section{Motion and Trace Photograph Correlation}

The different motions of the seal nosepiece can be illustrated by examining the trace photographs and referring to their position on figure 2 .

The trace photograph in figure 5 shows maximum amplitude on the accelerometer located at the $90^{\circ}$ position, which represents a 740-cps motion of 10 000-microinch amplitude. This is caused by a rocking about the $0^{\circ}$ to $180^{\circ}$ diameter.

The trace photograph in figure 6 shows that the accelerometer located in the $180^{\circ}$ position has the maximum amplitude. This trace represents a $1455-c p s$ motion of 1600 -microinch amplitude. This motion is also rocking about a diameter, in this case the $90^{\circ}$ to $270^{\circ}$ diameter.

In-phase axial accelerometer traces are shown in figure 7, representing diametral rocking about the $45^{\circ}$ to $225^{\circ}$ diameter.

The torsional motion is shown on the trace photograph in figure 8, which shows that the torsional accelerometer has the maximum amplitude. The torsional vibration frequency was $2800 \mathrm{cps}$ at a 600 -microinch displacement. This motion was not sensed by the axial accelerometers.

\section{Shifting Phase Relation}

The phase relation of the axial and torsional accelerometer traces is shown in figure 3. The trace photograph represents the sixth cycle of a longer trace photograph covering seven complete cycles.

In the case of severe diametral rocking ( $f i g .3$ ) the torsional trace is a combination of torsional oscillations and a component of the rocking motion acting in the same direction as the torsional component. For this reason the phase relation of the two axial and one torsional accelerometer is an indication of the seal nosepiece motions.

The two axial accelerometer traces shown in figure 3 are alternately in phase, then out of phase. The in-phase relation corresponds to rocking about the $45^{\circ}$ to $225^{\circ}$ diameter. The out-of-phase relation corresponds to rocking about the $135^{\circ}$ to $315^{\circ}$ diameter. Each revolution of the rocking diameter would be represented by two in-phase, out-of-phase cycles of the axial accelerometer traces. This means that an event time from 0.1096 to 0.1322 second is equivalent to one revolution of the rocking diameter.

The torsional trace in figure 3 does complete one cycle in 0.1096 to 0.1322 second. Thus, both the axial and torsional accelerometer traces are in 
agreement as to the period of one complete cycle.

The transient instability lasted $I / 2$ second and covered 27 cycles. The cycle rate was 43.6 cycles per second and corresponds very closely with the nominal shaft speed of $2500 \mathrm{rpm}$. The predominate frequency was $500 \mathrm{cps}$ at 100-g acceleration which is on the order of a 5000-microinch displacement. The pitch line frequency of the drive was $1290 \mathrm{cps}$ at $2500 \mathrm{rpm}$ and therefore was not a significant exciting influence.

The total indicator reading for the mating face runout was $500 \pm 100$ microinches. With a nominal shaft speed of $41.6 \mathrm{cps}$ the 500-cps, 5000-microinch displacement was not directly caused by mating face runout. Mating face runout can be reasonably assumed to have some exciting influence, however.

The wear on the carbon nosepiece is on the outside diameter, and cases of severe instability cause rapid wear. This excessive wear causes the nosepiece to assume a cone or convex shape. Figure 9 shows a graphic profile of a carbon nosepiece after a 38-minute test during which diametral rocking instability was encountered. A minimum of 0.004 inch of the original nose height was worn off during the test.

There were undoubtedly low-frequency axial vibrations caused by axial motion of the shaft and mating face runout. Because the accelerations were too small these motions were not evident in the trace photographs. At a shaft speed of $6000 \mathrm{rpm}$ a mating face runout of 1000 microinches would result in an acceleration of $0.5 \mathrm{~g}$. This is essentially $1 / 100$ to $1 / 10000$ the accelerations undcr discussion. These Iow-frequency, small accelerations are not responsible for the mechanical damage to the bellows face seals although these motions may have some effect in exciting the seals into more severe vibrations.

High-frequency (above $10 \mathrm{kcps}$ ) vibrations were recorded quite frequently during dynamic testing. These high-frequency vibrations averaged about $20 \mathrm{kcps}$, and had displacements of 1 to 10 microinches. Figure 10 shows vibrations of 20 kcps in the two axial and one torsional accelerometer traces. The highfrequency vibrations were being amplitude modulated to form the envelope patterns. Figure $I I(a)$ shows a characteristic trace without vibrations of 20 kcps, while figure $11(\mathrm{~b})$ taken 5 seconds later in real time shows a very similar trace with the superimposed $20 \mathrm{kcps}$ frequency vibrations.

When the seal was operating under stable conditions, the trace photograph is characteristic of that shown in figure 12. This trace picture shows relative calm on all three accelerometers with a very low-amplitude, medium-frequency vibration on the axial 2 accelerometer.

While the raw data were in the form of acceleration and vibrational frequency and could be plotted that way, it was more informative to plot displacement as a function of vibrational frequency and shaft speed. In that form the displacements ranged over five orders of magnitude.

The results of a test run with the undamped bellows face seal are shown in figure 13. Fifty-seven trace photographs were analyzed, and 132 data points were tabulated. Figure 13 represents the data with most of the plotted points 
being an average of several tabulated values. The bands of low-, medium-, and high-frequency vibrations are indicated and contain 92 percent of all the tabulated data points.

Figure 13 indicates the relation between the vibrational characteristics during periods of instability and the shaft speed. As the shaft speed increases, the high-frequency vibrations become less noticeable; that is, the increase in the displacement of the medium- and low-frequency vibrations tends to wash out the high-frequency vibrations in the trace photographs. Both the medium- and low-frequency vibrations increase in displacement as the shaft speed increases. The frequency bands are not a function of the shaft speed.

\section{Pressure Difference and Contact Load Effects}

The effect of different pressure differentials across the seal on the vibrational characteristics of the seal were examined. The test used a seal pressure balanced in such a manner that very little change in the contact pressure occurred when the pressure difference across the seal was changed. Figure 14 (a) shows the medium-frequency vibrations from 1 to $10 \mathrm{kcps}$ of the balanced seal at pressure differences of 20,60 , and 100 pounds per square inch and shaft speeds of 6000,8000 , and $10000 \mathrm{rpm}$.

Figure $14(b)$ also shows the results of a test on a seal in which the operating length of the seal was changed at constant pressure difference and shaft speed. By changing the operating length the contact load was changed by deflecting or compressing the bellows. The load increments were 1.0, 2.5, and 4.0 kilograms. These results are plotted for shaft speeds of 5000, 7500, and $10000 \mathrm{rpm}$.

\section{DISCUSSION OF RESULTS}

Considerable effort has been exerted by the manufacturers of bellows face seals to determine the natural frequencies of the bellows and to design them so that the bellows natural frequency will be above any operational vibration (refs. I and 2). While this is a necessary requirement, the results of this investigation show that the shaft speed and machine vibrations are not the primary cause of bellows seal vibrational instability.

The bellows seals under investigation showed a free vibration resonance of 120,500 , and $3000 \mathrm{cps}$. The 500-cps frequency was dominate and would correspond to a shaft speed of $30000 \mathrm{rpm}$. The $120-\mathrm{cps}$ resonance would correspond to a shaft speed of $7200 \mathrm{rpm}$. From this consideration and the knowledge that the total indicator reading for mating face runout was $500 \pm 100$ microinches, that the shaft axial movement was nominal, and that the noise level was moderate, satisfactory seal operation should have resulted at shaft speeds below $7200 \mathrm{rpm}$. However, the test results show that the operation is transitional at $2500 \mathrm{rpm}$, and transient periods of instability are established at $5000 \mathrm{rpm}$ and above.

It is of interest to note that the transient discussed in figure 2 has the primary resonance frequency of $500 \mathrm{cps}$, which was also indicated in the free vi- 
bration mode. This 500-cps frequency is higher than and not an obvious function of the $43.6-$ cps shaft speed.

From the results of changing pressure difference (fig. 14(a)) and changing axial load ( $\mathrm{fig}$. $14(\mathrm{~b})$ ) against vibrational frequency and shaft speed, some conclusions can be made. The most significant fact is that the vibrational characteristics of the bellows seal are not changed by either variable. Thus, the practice of pressure unbalancing a seal so that the interface load is increased in the hope of stabilizing the seal only results in wear, heat buildup, distortion, and excessive power loss in the seal.

The relation between axial displacement, acceleration, and vibrational frequency is shown in figure 15. Stable operation can be characterized by accelerations less than $10 \mathrm{~g}$. Unstable operation can be characterized by accelerations greater than $100 \mathrm{~g}$ with a transition zone between. There were few data points within the transition zone.

Transient instability can take place for only 1 second out of several minutes of operation. During the period of instability the seal leakage rate would increase, but during short-term transient instability the increase in the leakage rate would be very difficult to detect. For this reason, leakage rate excursions are not an accurate criteria in determining whether seal instability exists. The leakage rate increased during the tests as damage to the sealing surfaces was accumulated during periods of dynamic instability.

Noise in the form of a howl is an indication of severe instability. Excessive leakage and very short operation life can be expected under these conditions. Continuously unstable operation can cause failure of a bellows seal in only a few minutes of operation.

Transient instability is very difficult to detect in a normal bellows seal application. When unsatisfactory seal operation is encountered, an examination of the seal will be the best indication of unstable operation. These indications are wear and chipping of the seal nosepiece outside diameter, mechanical damage to the body parts of the seal from excessive motion or bouncing, and fatigue cracks of the bellows after short periods of operation.

It is apparent that phase change of the sealed fluid and friction forces generated in the interface are important exciting factors of dynamic instability in undamped bellows face seals.

\section{CONCLUSIONS}

From a study of the dynamic instability of undamped bellows face seals in a cryogenic liquid, the following conclusions were reached:

1. Undamped bellows face seals can operate in a dynamically unstable manner at shaft rotational frequencies below the seals resonant frequencies.

2. The effect of increasing the shaft rotational speed on the bellows face 
seal dynamic instability is to increase the magnitude of the vibrations but not change the vibrational frequencies of the instability.

3. Neither changes in the pressure difference across the bellows face seal nor normal loading due to spring force and unbalanced pressure difference affect the frequency of the dynamic instability.

4. The leakage rates are primarily a function of the accumulated damage to the seal due to operation under transient unstable conditions and not to periodic instability directly.

5. The predominate modes of motion of bellows face seals under dynamic instability are torsional oscillation and rocking about a diameter.

Lewis Research Center,

National Aeronautics and Space Administration, Cleveland, Ohio, October 27, 1965.

\section{REH'ERENCES}

1. Stevens, J. B.: Bellows-Type Axial Mechanical Seals. Machine Design - The Seals Book, Jan. 19, 1961, pp. 44-47.

2. Matt, Richard J.: High Temperature Metal Bellows Seals for Aircraft and Missile Accessories. J. Eng. Industry (ASME Trans.), ser. B, vol. 85, no. 3, Aug. 1963, pp. 281-287; Discussion, pp. 287-288.

3. Hart, Franklin D.; and Zorowski, Carl F.: Onset of Mechanical Separation in Bellows-Supported Rotary Face Seals. Paper No. 65-GTP-4, ASME, 1965. 


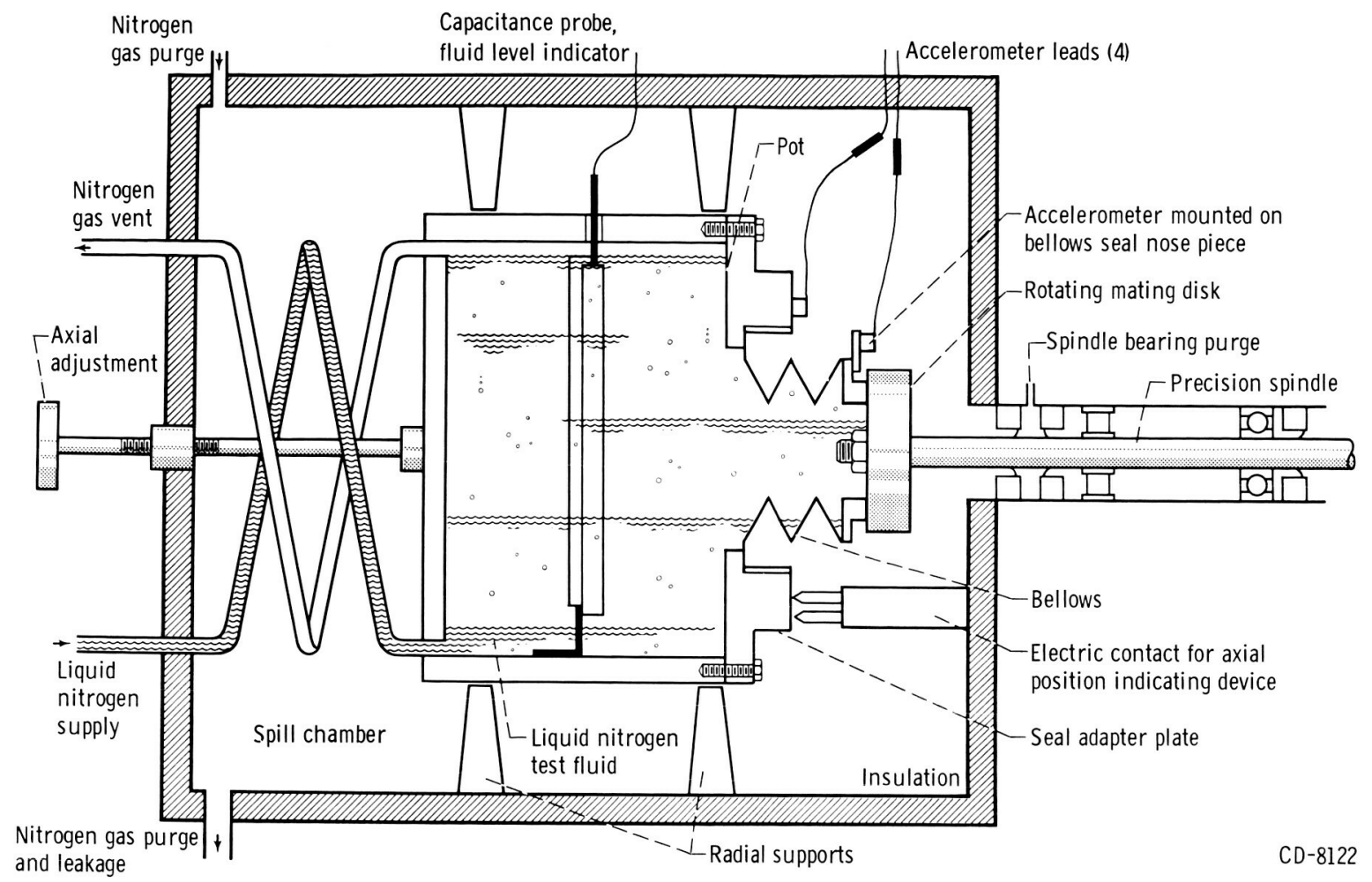

Figure 1. - Schematic of cryogenic seal test facility and seal test head.

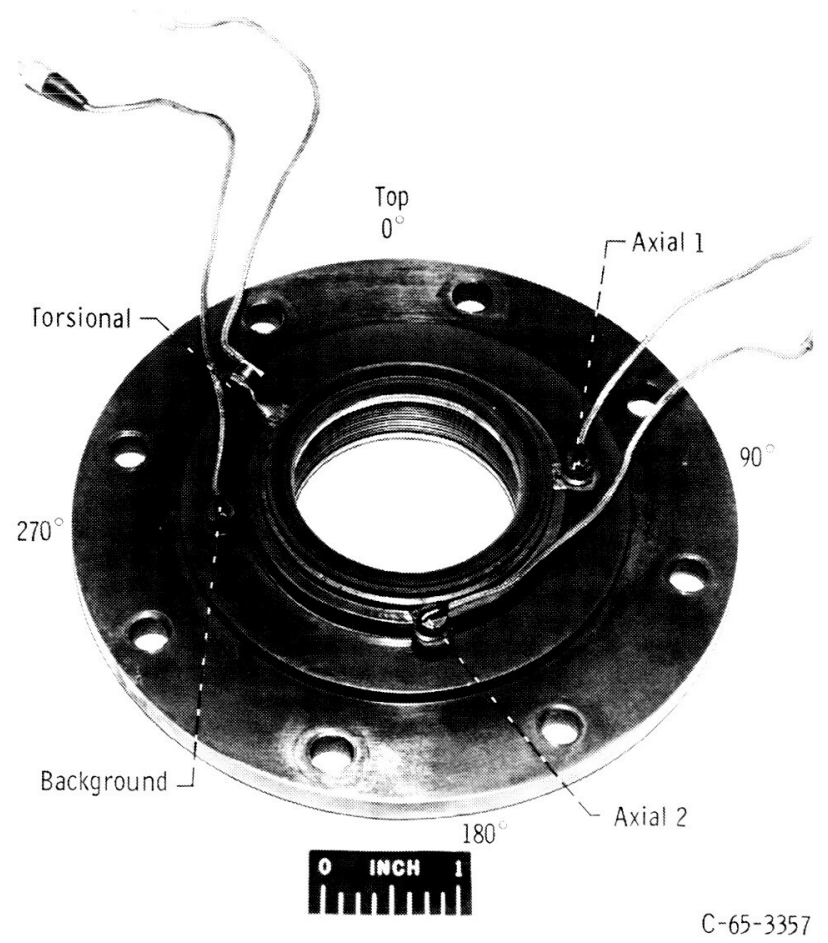

Figure 2. - Bellows face seal mounted in adapter plate with accelerometer mounting locations indicated. 


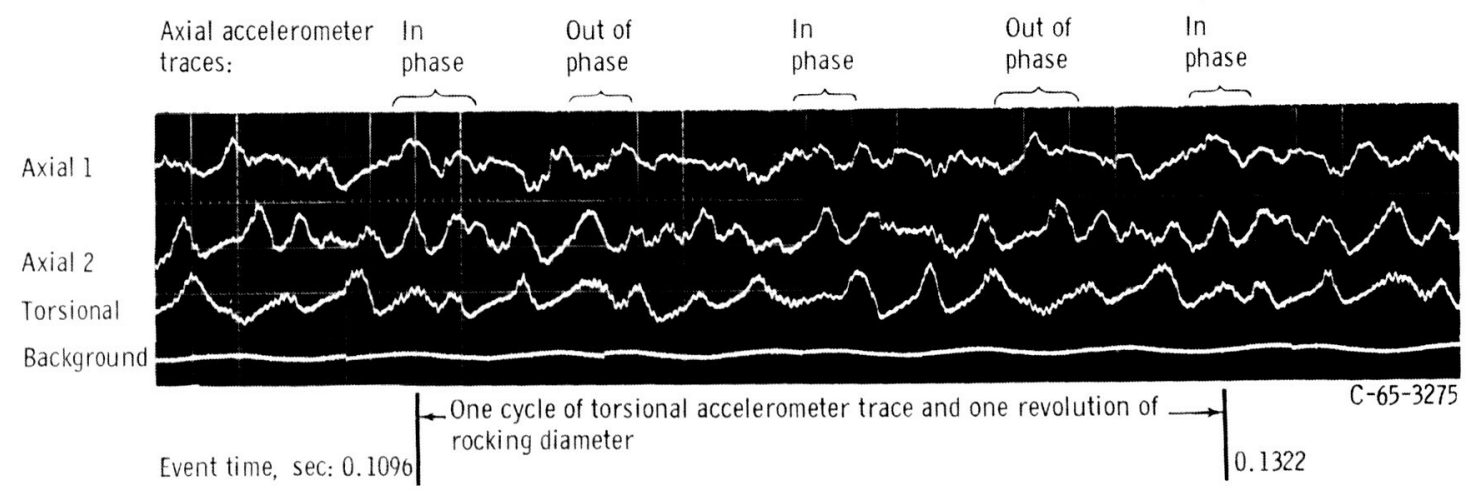

Figure 3. - Composit trace showing phase relations of diametral rocking about revolving diameter. 


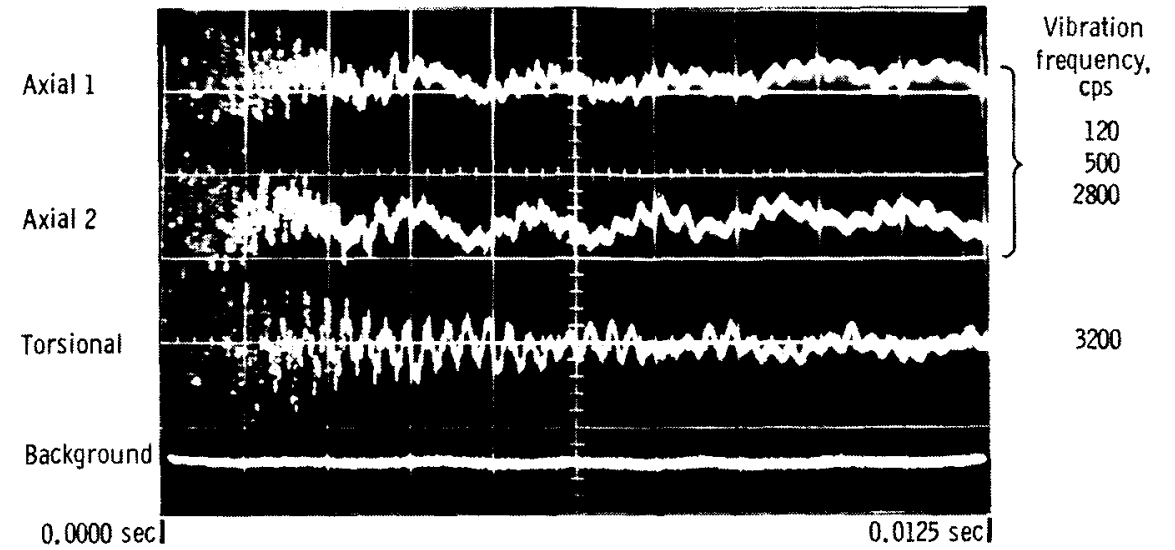

(a) Event time, 0.0000 to 0.0125 second.

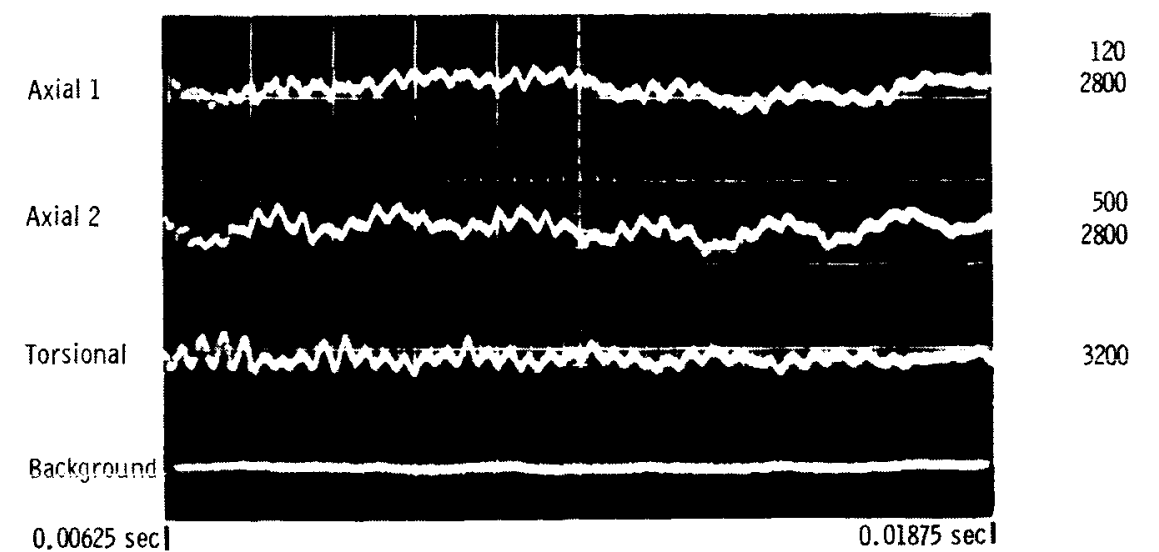

(b) Event time, 0.0625 to 0.01875 second after excitation.

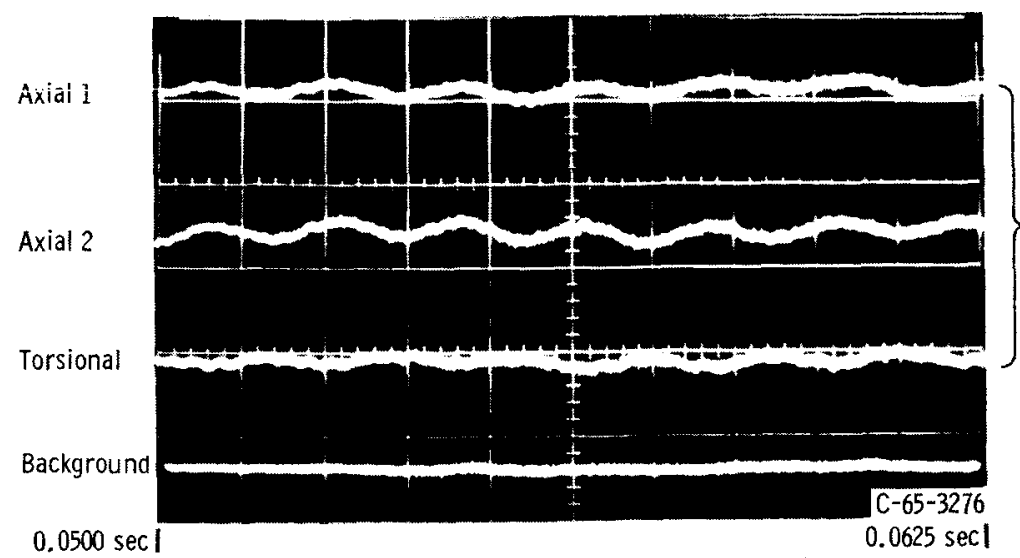

(c) Event time, 0.0500 to 0.0625 seconds after excitation.

Figure 4. - Natural resonant frequencies during free vibration. 

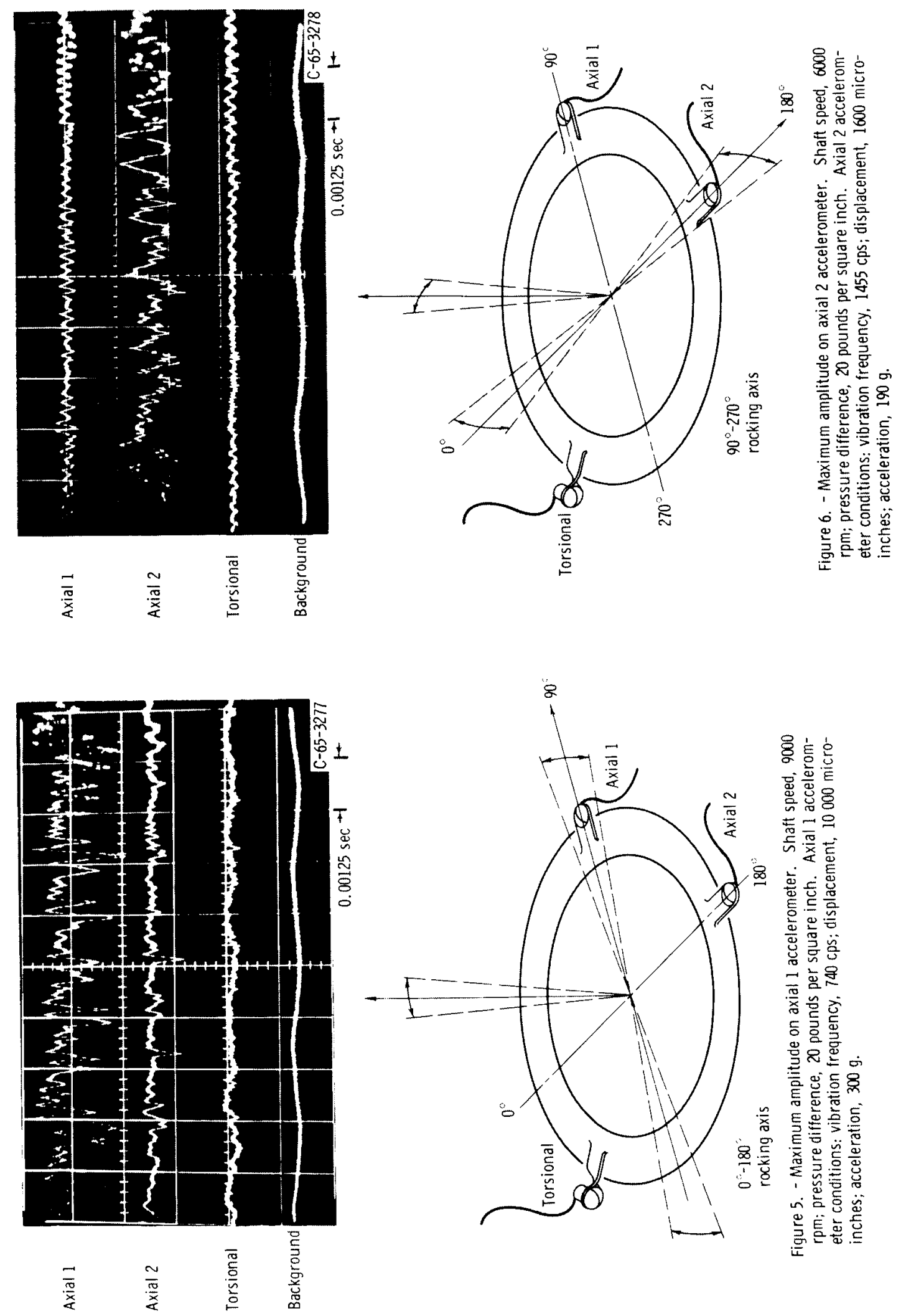

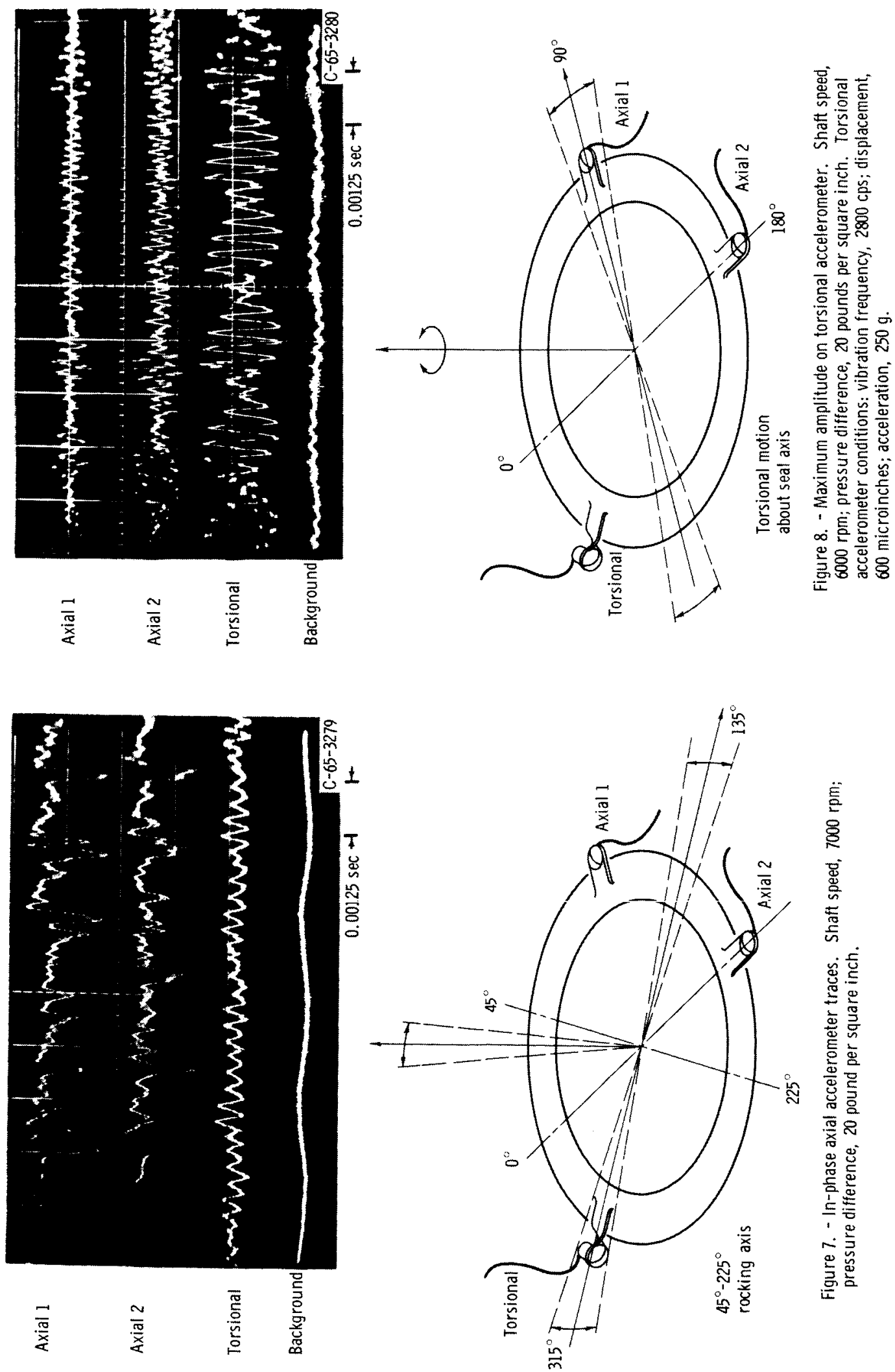


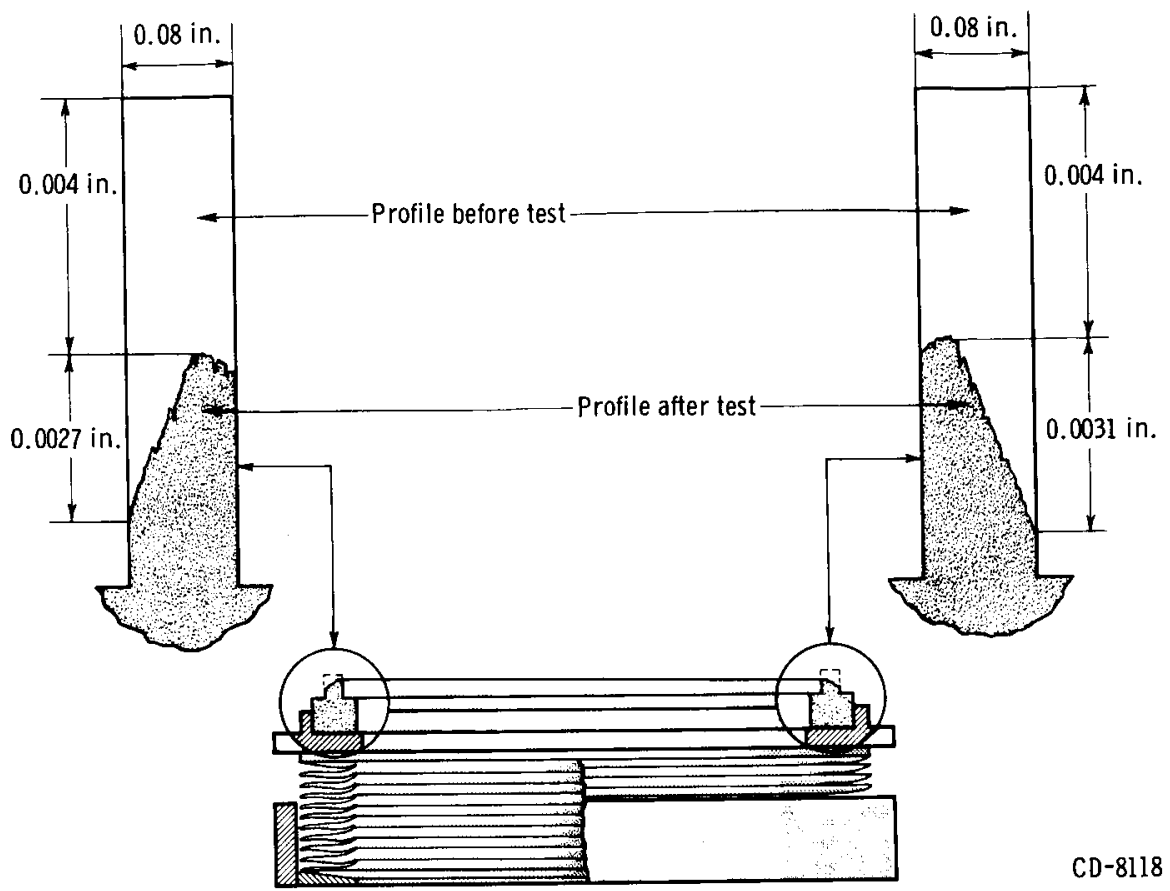

Figure 9. - Graphic profile of bellows face seal wear during 38-minute test in which diametral rocking was encountered.

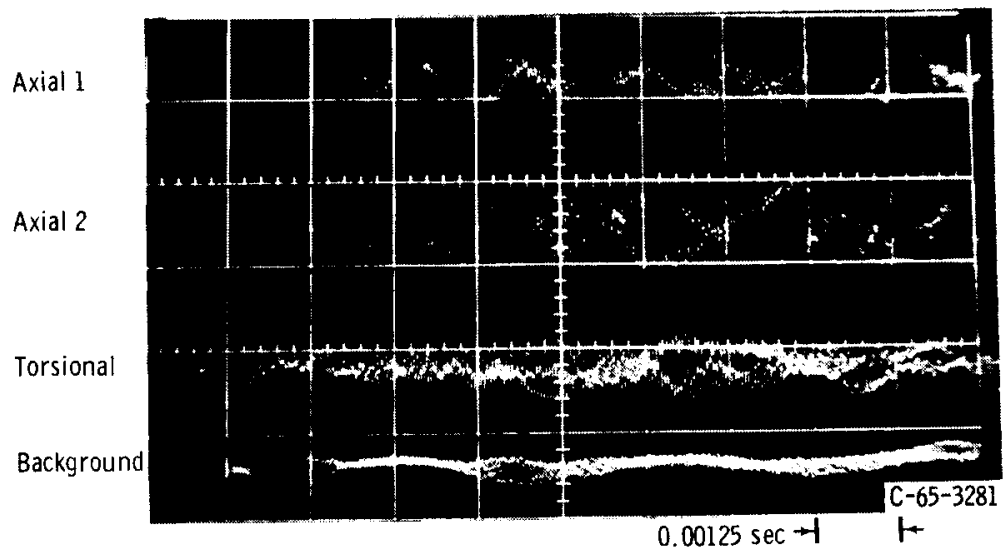

Figure 10. - Amplitude-modulated signal on all traces. Vibration frquency, 20 kcps; shaft speed. $2500 \mathrm{rpm}$; pressure difference, 20 pounds per square inch. 


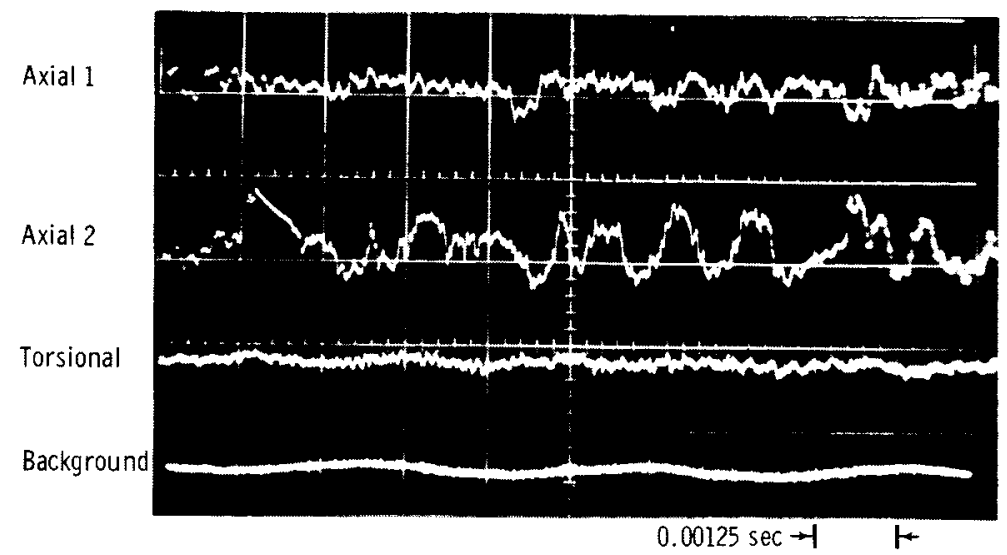

(a) Low-amplitude vibrations without high frequencies. Event time, 14 minutes 5 seconds.

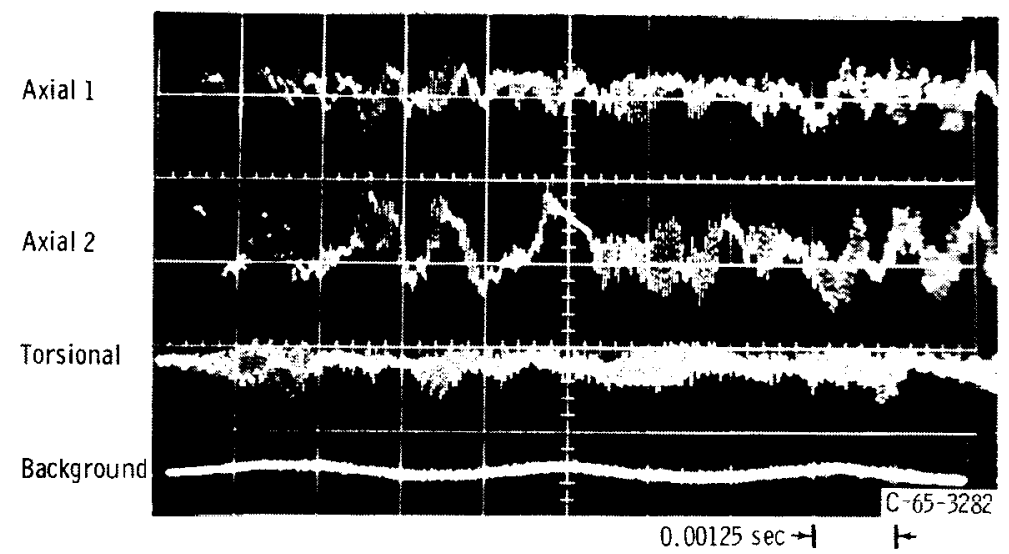

(b) Low-amplitude vibrations with high frequencies $(20 \mathrm{kcps})$. Event time, 14 minutes lô seconas.

Figure 11. - Superimposed high-frequency vibrations. Shaft speed, 5000 rpm; pressure difference, 20 pounds per square inch.

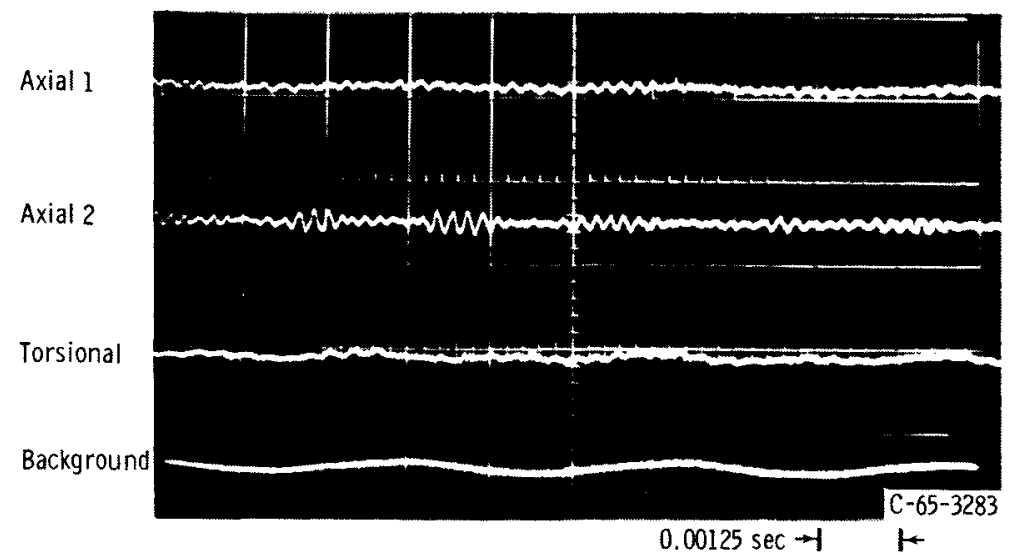

Figure 12. - Stable operation. Shaft speed, $9000 \mathrm{rpm}$; pressure difference, 20 pounds per square inch; vibration frequency, $4420 \mathrm{cps}$; displacement, 46 microinches; acceleration, $50 \mathrm{~g}$. 

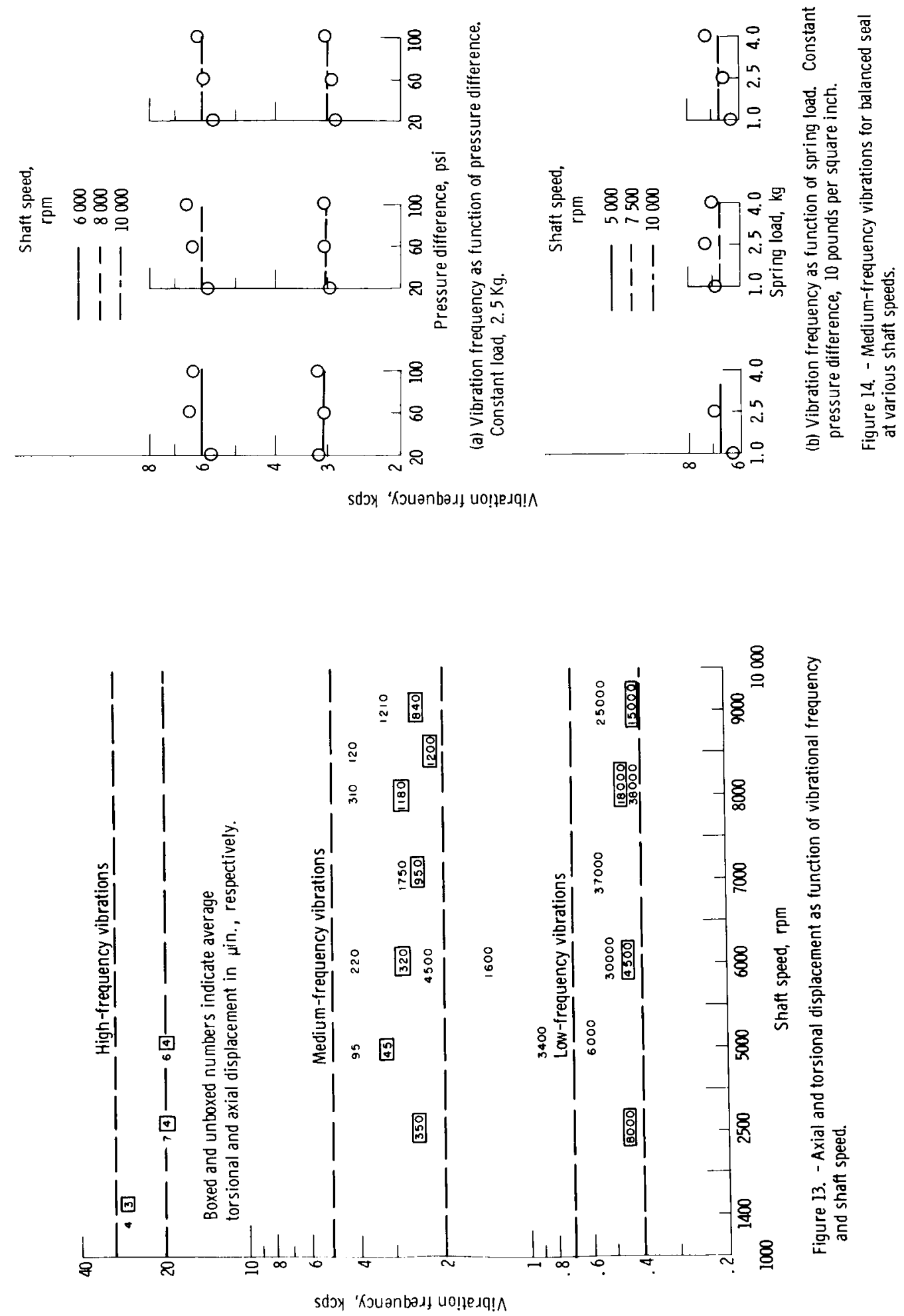


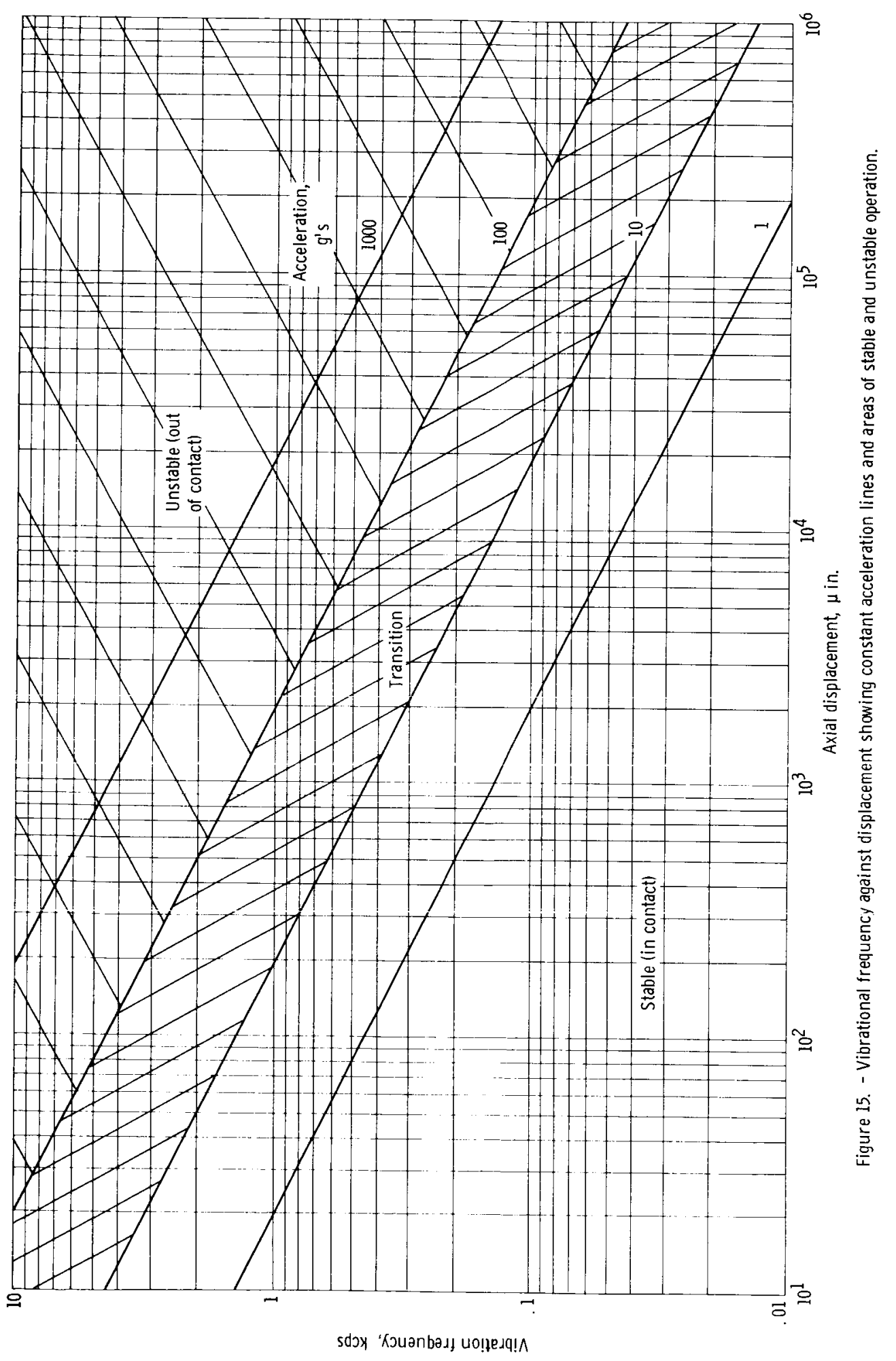

